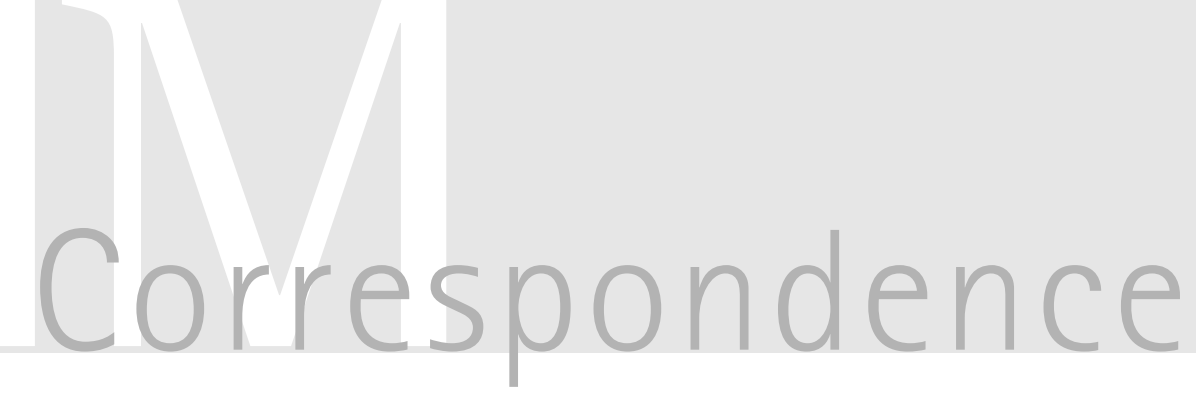

\title{
Undetected IktA genes within Fusobacterium necrophorum?
}

In a recent issue of this journal, Ludlam et al. (2009) outlined a PCR-based study on the basis of which they claimed that the $l k t A$ gene was not a universal virulence factor within strains of Fusobacterium necrophorum isolated from human and animal clinical cases. The authors also claimed that the detection of $l k t A$ was unevenly distributed across human and animal cases, between the two subspecies of F. necrophorum (subspecies necrophorum and subspecies funduliforme, hereafter referred to as Fnn and Fnf, respectively) and between the subspecies in isolates of bovine origin. We consider these unsafe conclusions as we are not convinced that the authors would have been able to detect the wide variety of $l k t A$ gene sequences that appear to be present within F. necrophorum with the PCR conditions they employed.

There are two reasons why we believe this is so, although these reasons are not completely unrelated. When using PCR on novel genetic material, it cannot be assumed that the primers will match the unknown target sequence, as even a single nucleotide mismatch at the $3^{\prime}$ end of either primer could completely stop amplification. As a result, the only conclusions that should ever be drawn from such diagnostic PCRs should be framed in terms of amplification or non-amplification, and not sequence presence or absence. To state absolutely that a sequence is not present is unjustifiable. Accordingly in our opinion the title of their paper ' $l k t A$-encoded leukotoxin is not a universal virulence factor in invasive Fusobacterium necrophorum infections in animals and man' (Ludlam et al., 2009) overstates the case on the evidence presented. This inability to prove the absence of something is not unique to PCRbased diagnostics, as most diagnostic techniques cannot definitively prove the absence of something. As a result when drawing conclusions from such diagnostic approaches, the wording used needs to reflect what can, and cannot, be proven.

We acknowledge that Ludlam et al. (2009) attempted to address the dangers of false negatives by using a positive control in the form of two geographically distant isolates of F. necrophorum that have had their $l k t A$ genes described (GenBank accession nos AF312861 and DQ672338). However, both of these isolates were from cattle, and recent studies suggest that the host may play a stronger influence on the genetic variation of $l k t A$ in F. necrophorum than geography, with sheep, goats and pigs tending to carry variants of $l k t A$ that are different to those found in cattle (Zhou et al., 2009a, 2010). Additionally, the Fnn lktA sequence isolated from cattle in the USA (GenBank accession no. AF312861) and the partial sequence isolated from cattle in New Zealand (Zhou et al., 2009a) are identical.

When we aligned the primer and probe sequences reported by Ludlam et al. (2009) with the published $F$. necrophorum lktA sequences (GenBank accession nos
AF312861, AY972049 and FJ230830-

FJ230832) in a region where variation has been reported previously (Zhou et al., 2009a), mismatches were found between their sequences and some of the $F$. necrophorum lktA sequences, including three from cloned DNA derived from uncultured strains of F. necrophorum (Fig. 1). In particular, there are at least 1, 3 and 1 nucleotide mismatches in the forward, reverse and probe-binding regions, respectively, when compared to $F$. necrophorum variants $\mathrm{C}$ and $\mathrm{D}$, the former being the most common F. necrophorum variant found in sheep, goats and pigs (Zhou et al., 2009a, 2010). Mismatches in these regions might prevent these variants from being amplified and hence these variants of the $l k t A$ gene may go undetected. Therefore, it is also possible that the primers and probes reported by Ludlam et al. (2009) might not detect all $F$. (a)

LT2 forward:
Fnn:
Fnf:
Variant B:
Variant C:
Variant D:

(b)

$$
\begin{aligned}
& \text { LT2 reverse: } \\
& \text { Fnn: } \\
& \text { Fnf: } \\
& \text { Variant B: } \\
& \text { Variant C: } \\
& \text { Variant D: }
\end{aligned}
$$
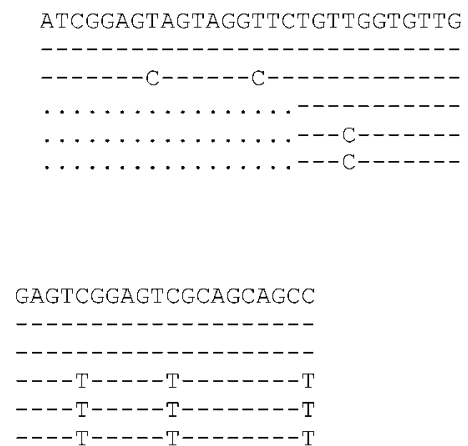

(c)

LT2 probe:
Fnn:
Fnf:
Variant B:
Variant C:
Variant D:

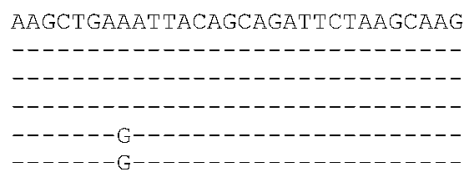

Fig. 1. Alignments of the primer and probe sequences reported by Ludlam et al. (2009) with F. necrophorum IktA sequences: (a) the LT2 forward primer, (b) the LT2 reverse primer and (c) the LT2 probe binding region. Dashes represent nucleotide identical to the top (primer/probe) sequence, and unknown nucleotide sequences are shown by dots. All sequences are presented $5^{\prime}$ to $3^{\prime}$, and the reverse complementary sequence of LT2 reverse primer is shown. The GenBank accession numbers for the $F$. necrophorum IktA sequences are AF312861 (Fnn), AY972049 (Fnf), FJ230830 (variant B), FJ230831 (variant C) and FJ230832 (variant D). 
necrophorum variants, which may explain why they found only two sequences corresponding to the reported isolates of Fnn and Fnf.

Interestingly, although we have previously reported a total of four variants of $F$. necrophorum from sheep, goats, cattle and pigs, there was no evidence for the presence of Fnf in these animal species (Zhou et al., 2009a, 2010), while in the paper by Ludlam et al. (2009) Fnf appears to be common in animals, and even dominant over Fnn in some animal species such as cattle and sheep.

The issue of substantive variation in $l k t A$ genes preventing PCR techniques from being utilized to detect fusobacteria has been noted by other authors (Tadepalli et al., 2008). They reported that Fusobacterium equinum (a bacterium phenotypically similar to $F$. necrophorum) produces a leukotoxin of equivalent size and potency to that of F. necrophorum, and that it is encoded by an $l k t A$ gene (shown to be present using probes and Southern hybridization), but that no amplicons were produced from the nominal $l k t A$ gene of $F$. equinum using PCR, probably because of sequence differences between the $l t k A$ genes of F. equinum and the two F. necrophorum subspecies. A portion of the $l k t A$ gene of $F$. equinum was only recently sequenced (Zhou et al., 2009b) and this confirmed that the gene was substantively different at the DNA sequence level from both Fnn and Fnf.

While we agree with the conclusion drawn by Ludlam et al. (2009) that vaccines against the $l k t A$ gene of Fnn may be unsuitable to be used against all strains, we do not agree with their reasoning and the assumptions that their conclusions are based on. Rather than $l k t A$ vaccines being likely to fail due to $l k t A$ not being universally present in all $F$. necrophorum strains, we consider it a possibility that vaccines could fail due to genetic differences observed in the $l k t A$ genes found in the various $F$. necrophorum strains or other F. necrophorum-like bacteria.

\section{Grant Bennett, Huitong Zhou and Jon G. H. Hickford}

Department of Agricultural Science, Faculty of Agriculture and Life Sciences, PO Box 84, Lincoln University, Lincoln 7647, New Zealand
Correspondence: Jon G. H. Hickford (jon.hickford@lincoln.ac.nz)

Ludlam, H. A., Milner, N. J., Brazier, J. S., Davies, I. H., Perry, K., Marriott, R. K., Donachie, L. \& Curran, M. D. (2009). lktAencoded leukotoxin is not a universal virulence factor in invasive Fusobacterium necrophorum infections in animals and man. J Med Microbiol 58, 529-530.

Tadepalli, S., Stewart, G. C., Nagaraja, T. G., Jang, S. S. \& Narayanan, S. K. (2008).

Fusobacterium equinum possesses a leukotoxin gene and exhibits leukotoxin activity. Vet Microbiol 127, 89-96.

Zhou, H., Bennett, G. \& Hickford, J. G. (2009a). Variation in Fusobacterium necrophorum strains present on the hooves of footrot infected sheep, goats and cattle. Vet Microbiol 135, 363-367.

Zhou, H., Bennett, G., Kennan, R. M., Rood, J. I. \& Hickford, J. G. (2009b). Identification of a leukotoxin sequence from Fusobacterium equinum. Vet Microbiol 133, 394-395.

Zhou, H., Dobbinson, S. \& Hickford, J. G. H. (2010). Fusobacterium necrophorum variants present on the hooves of lame pigs. Vet Microbiol (in press). doi: 10.1016/

j.vetmic.2009.09.014

DOI 10.1099/jmm.0.016709-0

\section{Authors' reply to 'Undetected IktA genes within Fusobacterium necrophorum?': presence or absence of an amplicon - the cornerstone of molecular diagnostics}

Bennett et al. (2010) raise some interesting points and present some intriguing sequence data for the leukotoxin gene (lktA) of Fusobacterium necrophorum, drawn from work recently published by them, indicating three novel variants in the lktA gene (Zhou et al., 2009a). Bennett et al. (2010) speculate from these data that the conclusions drawn in our previous communication (Ludlam et al., 2009), in which we reported our failure to detect the gene employing PCR in a significant proportion of the two subspecies of $F$. necrophorum (subspecies necrophorum and funduliforme) recovered from infections in humans and animals (Ludlam et al., 2009), may be unsound.

Whilst we accept that a limitation of PCRbased diagnostics can be the failure to detect a novel variant of a pathogen, we cannot accept the assertion of Bennett et al. (2010) that the only conclusion that should ever be drawn from negative diagnostic PCR results is simply non-amplification of the sequence in question, rather than absence of the gene, or host organism. Following rigorous commercial and in-house test development and validation of PCR-based diagnostics of the sort we reported, diagnosis and management of infectious diseases is now routinely based on both positive and negative PCR results. Indeed, we note that the authors themselves ruled out the presence of $F$. necrophorum subspecies funduliforme in material from the lesions of the animal hooves that they examined on precisely this basis; i.e. a negative PCR result for $l k t A$ (Zhou et al., 2009a).

We were aware of the possible existence of sequence variation and went to great lengths to ensure that putative variants of the sort described by Zhou et al. (2009a) would not pass undetected. Whilst Bennett et al. (2010) focus on the LT2 primer set and TaqMan probe that emphasize their novel sequence data, they do not mention the other two primer sets (LT1 and LT3) that we employed, which targeted entirely different regions of the three published entire $l k t A$ gene sequences (F. necrophorum subspecies necrophorum, AF312861 and DQ672338; F. necrophorum subspecies funduliforme, AY972049). Our LT1 primer set is perfectly matched for all three known entire $l k t A$ gene sequences, while the LT3 forward primer contained just a single mismatch with

F. necrophorum subspecies funduliforme AY972049 at the $5^{\prime}$ end of the primer and the LT3 reverse primer was perfectly matched for all three. All three primers sets amplified the $l k t A$ gene from the two 